\title{
Analysis and numerical approximation of viscosity solutions with shocks: application to the plasma equation
}

\author{
Susana Serna \\ serna@mat.uab.es \\ Departament de Matematiques, Universitat Autonoma de Barcelona, 08193 Bellaterra, Spain
}

\begin{abstract}
.
We consider a new class of Hamilton-Jacobi equations arising from the convective part of general Fokker-Planck equations ruled by a non-negative diffusion function that depends on the unknown and on the gradient of the unknown. The new class of Hamilton-Jacobi equations represents the propagation of fronts with speed that is a nonlinear function of the signal. The equations contain a nonstandard Hamiltonian that allows the presence of shocks in the solution and these shocks propagate with nonlinear velocity. We focus on the one-dimensional plasma equation as an example of the general Fokker-Planck equations having the features we are interested in analyzing. We explore features of the solution of the corresponding Hamilton-Jacobi plasma equation and propose a suitable fifth order finite difference numerical scheme that approximates the solution in a consistent way with respect to the solution of the associated Fokker-Planck equation. We present numerical results performed under different initial data with compact support.
\end{abstract}

Keywords: Fokker-Planck equation, Hamilton-Jacobi equations, plasma equation, numerical schemes PACS: $02.70 . \mathrm{Bf} ; 02.60 . \mathrm{Cb} ; 52.35 . \mathrm{Tc}$

\section{INTRODUCTION}

Classical Fokker-Planck equations represent a wide class of anisotropic diffusion equations that model transport by diffusion of a physical magnitude in a continuum medium $([1,2,3,11])$. Solutions of these equations contain diffusion fronts that propagate with finite speed. A Fokker-Planck equation can be written in conservation form as

$$
u_{t}=\operatorname{div}(g(u,|\nabla u|) \nabla u)
$$

where $g(u, p)$ is a non-negative real function defined for $u \geq 0$.

Diffusion equations of the form (1) might allow fronts (jump discontinuities) in their solutions and we are interested in analyzing the terms responsible of their formation. In order to analyze the convective part of the Fokker-Planck equation we expand spatial derivatives of the flux equation (1) as

$$
u_{t}=\frac{\partial g}{\partial u}|\nabla u|^{2}+g(u,|\nabla u|) \Delta u+\frac{\partial g}{\partial p} \frac{L(\nabla u)}{|\nabla u|}
$$

where

$$
L(\nabla u)=u_{x}^{2} u_{x x}+2 u_{x} u_{y} u_{x y}+u_{y}^{2} u_{y y}
$$

in two spatial dimensions.

As it is proven in [9], diffusion fronts propagate according to a hyperbolic conservation law and their dynamics is ruled by the convective part of (1) which is the first term in (2). The Hamilton-Jacobi equation associated to this term is

$$
u_{t}=\frac{\partial g}{\partial u}|\nabla u|^{2}
$$

which results from removing diffusions terms in (2).

In the following sections we consider the plasma equation as an example of a Fokker-Planck equation where diffusion fronts are generated. We show evidence of the presence of jumps in the solution of the plasma equation as a consequence of the formation of shocks in the solution of the associated Hamilton-Jacobi plasma equation. We present numerical results supporting our study. 


\section{PLASMA EQUATION}

In this section we consider the one dimensional Fokker-Planck plasma equation ([8]),

$$
u_{t}=\left(\frac{u^{5 / 2} u_{x}}{1+u\left|u_{x}\right|}\right)_{x}
$$

with $t \in] 0, T], u \geq 0, x \in[0,1]$, under homogeneous Neumann boundary conditions at $x=0$ and $x=1$ and using initial data consisting of real functions defined in $[0,1]$ with compact support in $] 0,1[$.

We can express plasma equation (5) in non-divergence form by calculating derivatives in an explicit form as in (2),

$$
u_{t}=\frac{3}{2} u^{3 / 2} \frac{\frac{5}{3}+u\left|u_{x}\right|}{\left(1+u\left|u_{x}\right|\right)^{2}} u_{x}^{2}+\frac{u^{5 / 2}}{\left(1+u\left|u_{x}\right|\right)^{2}} u_{x x}
$$

Then, the Hamilton-Jacobi equation associated to the convective term of the plasma equation is

$$
u_{t}=\frac{3}{2} u^{3 / 2} \frac{\frac{5}{3}+u\left|u_{x}\right|}{\left(1+u\left|u_{x}\right|\right)^{2}} u_{x}^{2}
$$

We name equation (7) Hamilton-Jacobi plasma equation.

Let us perform a similar heuristic asymptotic analysis as the one presented in [9] for the relativistic heat equation to understand the behavior of the plasma equation (5) near jump discontinuities

Around possible jumps in the solution where $\left|u_{x}\right|>>1$ the flux in (5) approaches to

$$
\frac{u^{5 / 2} u_{x}}{1+u\left|u_{x}\right|} \rightarrow u^{3 / 2} \operatorname{sgn}\left(u_{x}\right)
$$

which means that around diffusion fronts equation (5) resembles a non-linear hyperbolic conservation law with flux $u^{3 / 2} \operatorname{sgn}\left(u_{x}\right)$, i.e.

$$
u_{t} \approx\left(u^{3 / 2} \operatorname{sgn}\left(u_{x}\right)\right)_{x}
$$

On the other hand we can state that shock waves are ruled by Hamilton-Jacobi equation (7) as it approaches to

$$
u_{t} \approx \frac{3}{2} u^{1 / 2} \operatorname{sgn}\left(u_{x}\right) u_{x}
$$

when $\left|u_{x}\right|>>1$ (around jumps). Thus, characteristics intersect in (7) for a non-constant initial data and shocks may arise in finite time. Let us remark that this Hamiltonian depends on $u$ in a nonstandard way from the point of view of classical theory of viscosity solutions $([4,5,6])$.

From this analysis we can state that the convective term of the plasma equation approaches equation (10) around jump discontinuities and is responsible of the development of shocks in the solution of the plasma equation (5). Shock waves are allowed at the boundary of the support of the so-called entropy solution expanding the support according to the velocity prescribed by the Rankine-Hugoniot condition ([7]) with speed depending on $u$.

\section{NUMERICAL APPROXIMATION AND EXAMPLES}

In this section we propose a finite difference numerical scheme to approximate the Hamilton-Jacobi equation (7) and present a set of numerical experiments to show numerical evidence of our analysis. We compare in a consistent way the solutions for the Hamilton-Jacobi plasma equation with the solutions of the associated Fokker-Planck equation.

For the approximation of the solution of the plasma Fokker-Planck equation we use the conservative finite difference numerical scheme proposed in [9]. The explicit numerical scheme is consistent, stable, conserves mass exactly, preserves positivity and satisfies a discrete local maximum principle as demonstrated in [9]. The numerical method is combined with the Weighted PowerENO reconstruction procedure ([12]) to reach fifth order accuracy in space and the third order TVD Runge-Kutta procedure ([14]) for the integration in time. For the approximation of the solution of the Hamilton-Jacobi plasma equation we use a local Lax-Friedrichs numerical scheme. 
Let us consider a one-dimensional Hamilton-Jacobi equation

$$
u_{t}+H\left(u, u_{x}\right)=0 \text { where } H(u, p):=-G\left(u, \sqrt{p^{2}}\right) p^{2}
$$

We define the computational domain as follows. We consider a uniform mesh in the interval $[0,1]$ in $\mathrm{N}$ partitions of length $\Delta x=\frac{1}{N}$. We denote by $u_{j}^{n}$ the numerical approximation of $u\left(x_{j}, t_{n}\right)$ where $x_{j}$ is the $j$-node of the interval and $t^{n}=n \Delta t$ where $\Delta t$ is the time step size, $\Delta t>0$.

A first order finite differences numerical scheme to solve (11) is defined as

$$
u_{j}^{n+1}=u_{j}^{n}-\Delta t \tilde{h}\left(u_{j}^{n}, \frac{\Delta_{-}^{x} u_{j}^{n}}{\Delta x}, \frac{\Delta_{+}^{x} u_{j}^{n}}{\Delta x}\right)
$$

where $\tilde{h}$ is a Lipschitz, monotone and consistent numerical Hamiltonian, $\tilde{h}(u, p, p, q, q)=H(u, p, q)$ and

$$
\Delta_{-}^{x} u_{j}^{n}=u_{j}^{n}-u_{j-1}^{n} ; \quad \Delta_{+}^{x} u_{j}^{n}=u_{j+1}^{n}-u_{j}^{n}
$$

The Local Lax-Friedrichs Hamiltonian is defined as

$$
\tilde{h}^{L L F}\left(u, p^{-}, p^{+}\right)=H\left(u, \frac{p^{-}+p^{+}}{2}\right)-\frac{\alpha_{1}}{2}\left(p^{+}-p^{-}\right)
$$

where

$$
\alpha_{1}=\max \left|H_{p}(u, p, q)\right|, \quad H_{p}=\frac{\partial H}{\partial p}
$$

and the maxima are taken on the local interval $p \in I\left(p^{-}, p^{+}\right) ; I(a, b)=[\min (a, b), \max (a, b)]$ overall $u$ in the domain.

The extension to high order accuracy is obtained from the basic first order scheme by computing high-order approximations to $u^{-}=\Delta_{-}^{x} u_{j}^{n}$ and $u^{+}=\Delta_{+}^{x} u_{j}^{n}$ by means of a reconstruction procedure ([10]). We use the Weighted PowerENO fifth order reconstruction procedure to reach fifth order of accuracy in space. The implementation process of this procedure for Hamilton-Jacobi equations is detailed in [13]. High order accuracy in time is obtained by using an explicit third order TVD Runge-Kutta scheme ([14]).

\section{Example 1: Double step initial data}

We approximate the solution of the Fokker-Planck and the Hamilton-Jacobi plasma equations for the double step initial data

$$
u_{0}(x)= \begin{cases}2 & 1 / 3 \leq x \leq 2 / 3 \\ 1 & 1 / 6 \leq x<1 / 3 \\ 1 & 2 / 3 \leq x \leq 5 / 6 \\ 0 & \text { elsewhere }\end{cases}
$$

in $x \in[0,1]$ under homogeneous Neumann boundary conditions. We compute the solution at time $t=0.1$ with 200 grid points. Figure 1 displays the results for both equations. We observe that the numerical schemes behave robust, stable and accurate and the numerical approximations are consistent with the dynamics ruled by the conservative flux and the Hamiltonian respectively. The initial discontinuities propagate with correct speed for the Hamilton-Jacobi case and the fronts propagate according to the Rankine-Hugoniot conditions for the plasma equation.

\section{Example 2: Continuous data} data

We approximate the solution of the Fokker-Planck and the Hamilton-Jacobi plasma equations for a continuous initial

$$
u_{0}(x)=160 \max \left(0.0625-(x-0.5)^{2}, 0\right)
$$

in $x \in[0,1]$ under homogeneous Neumann boundary conditions. We compute the solution until $t=0.1$ with 200 grid points. Figure 2 displays the results for both equations. We observe that diffusion fronts generated at the boundary of the solution for the Fokker-Planck case propagate with the same speed as the shock waves generated in the solution of the Hamilton-Jacobi plasma equation. The proposed numerical scheme for the Hamilton-Jacobi plasma equation captures the formation and evolution of shock waves in a sharp, accurate and stable way. 

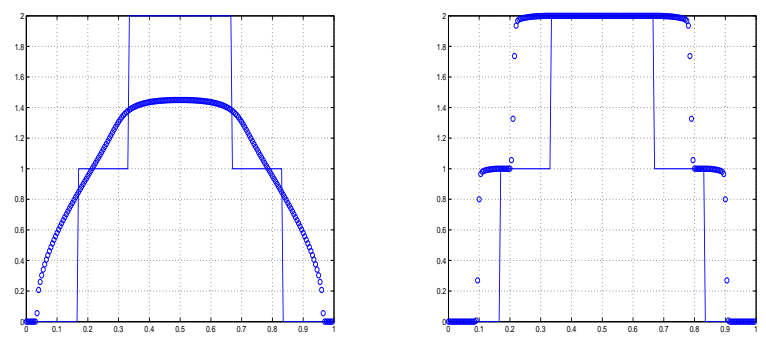

FIGURE 1. Example 1. Evolution at time $t=0.1$, "o" numerical approximation versus "-" initial data. Left, Fokker-Planck plasma equation. Right, Hamilton-Jacobi plasma equation
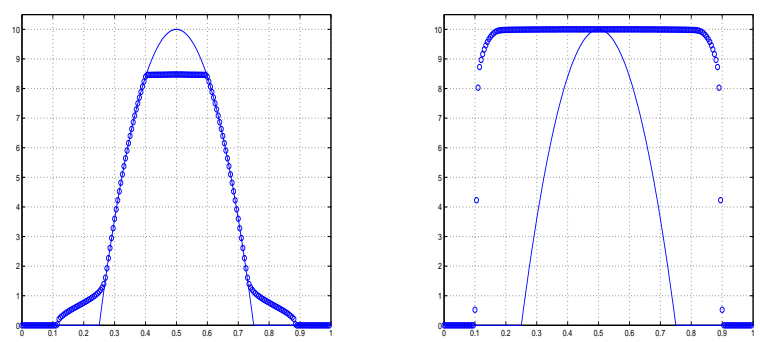

FIGURE 2. Example 2. Evolution at time $t=0.1$, "o" numerical approximation versus "-" initial data. Left, Fokker-Planck plasma equation. Right, Hamilton-Jacobi plasma equation

\section{CONCLUSIONS}

We analyze a class of Hamilton-Jacobi equations arising from the convective part of general Fokker-Planck equations describing the propagation of fronts with speed that is nonlinear function of the signal. We consider the plasma equation as an example of a Fokker-Planck equation where diffusion fronts are generated and show evidence of the presence of jumps in the solution of the plasma equation as a consequence of the formation of shocks in the solution of the associated Hamilton-Jacobi plasma equation. We propose a fifth order finite difference numerical scheme that approximates the solution of the Hamilton-Jacobi equation consistently with respect to the solution of the associated Fokker-Planck equation. We present numerical results supporting our study.

\section{ACKNOWLEDGMENTS}

The author acknowledges support by MICINN projects "Programa Ramón y Cajal" and MTM2008-03597.

\section{REFERENCES}

1. F. Andreu, V. Caselles, JM Mazon and S. Moll, Arch. Ration. Mech. Anal. 182, 269-297 (2006).

2. F. Andreu, V. Caselles and JM Mazon, J. Differential Eqn. 245, 3639-3663 (2008).

3. V. Caselles, J. of Diff. Eqns 250 3311-3348 (2011).

4. M. Crandall, H. Ishii, P. Lions, Bull. Amer. Math. Soc., 27 1-67, (1992).

5. M. Crandall and P. Lions, Trans. Amer. Math. Soc., 277, 1-42 (1983).

6. M. Crandall and P. Lions, Math. Comput, 43, 1-19 (1984).

7. R. Courant, K. Friedrichs, H. Lewy, Math. Ann., 100 32-74, (1928).

8. J.J. Duderstadt and G.A. Moses, Inertial Confinement Fusion, John Wiley and Sons (1982).

9. A. Marquina, J. Comput. Phys., 229 2659-2674 (2010)

10. S. J. Osher and C-W. Shu ,SIAM J. Numer. Anal., 28, 907-922 (1991).

11. P. Rosenau, Phys. Rev. A 46 7371-7374 (1992).

12. S. Serna, A. Marquina, J. Comput. Phys, 194 632-658, (2004).

13. S. Serna and J. Qian. J. Sci. Comp. 29 57-81, (2006)

14. C.W. Shu, S Osher, J. Comput. Phys., 83 32-78 (1989). 